\title{
Agôn
}

Revue des arts de la scène

1 | 2008

Interstices, entractes et transitions

\section{Eccyclème et transition spatiale dans le théâtre tragique grec du Ve siècle av. J.C.}

\section{Anne-Sophie Noel}

\section{(2) OpenEdition \\ Journals}

Édition électronique

URL : http://journals.openedition.org/agon/710

DOI : 10.4000/agon.710

ISSN : 1961-8581

Éditeur

Association Agôn

Référence électronique

Anne-Sophie Noel, «Eccyclème et transition spatiale dans le théâtre tragique grec du Ve siècle av.

J.C. », Agôn [En ligne], 1 | 2008, mis en ligne le 18 décembre 2008, consulté le 30 mai 2020. URL :

http://journals.openedition.org/agon/710 ; DOI : https://doi.org/10.4000/agon.710 


\title{
Eccyclème et transition spatiale dans le théâtre tragique grec du V siècle av. J.C.
}

\begin{abstract}
À Athènes au V $\mathrm{V}^{\mathrm{e}}$ siècle av. J.-C., l'espace du théâtre de Dionysos est structuré par des contraintes fortes, d'ordre physique aussi bien que culturel : parmi elles, la division stricte de l'espace dramatique en une partie visible et une autre invisible. L'espace scénique visible, se déployant dans les limites de l'orchestra, représente toujours un espace public ouvert - très souvent la cité ou bien encore le camp des Grecs au pied de Troie. L'espace invisible se confond avec le hors-scène, qui recouvre deux lieux distincts : un espace virtuel extérieur, s'étendant au-delà de l'espace scénique, dont l'identité n'est pas fixe d'un drame à l'autre, et auquel conduisent les deux entrées latérales nommées parodos; un espace virtuel intérieur situé au-dedans de la skénè. Ce bâtiment de scène en bois servait à l'origine à stocker l'ensemble des accessoires théâtraux; investie d'une fonction dramatique, la skénè devient palais, demeure ou encore baraque, et dans le corpus qui nous est parvenu, la plus ancienne attestation de son utilisation dramatique remonte à la création de 1'Orestie d'Eschyle en 458. Comme son équivalent réel dans la société athénienne classique, l'espace domestique ou oikos est un espace tenu secret, fermé et caché, vie privée et publique se développant sur des terrains strictement séparés.
\end{abstract}

Ces deux espaces extra-scéniques échappent à la vue des spectateurs : ils sont les territoires de l'imaginaire. Le spectateur en perçoit des bribes, à travers les paroles des personnages : celles d'un messager sortant du palais, pour raconter comment un meurtre ou un suicide s'est produit. Mais jamais un intérieur n'est décrit, jamais un paysage n'est dépeint, seuls les détails qui jouent un rôle particulier dans l'action ou la caractérisation d'un personnage peuvent être glissés à dessein dans un récit. Ces lieux non visibles sont en partie créés pour y situer des actions ne devant pas ou ne pouvant pas êtres vues : les actions violentes - sacrifices, meurtres, suicides - exclus de l'aire de jeu visible par respect des convenances, mais sans doute également pour des raisons techniques. En plus d'être difficile à jouer, une scène de meurtre par exemple, pose le problème de l'évacuation du mort et du changement rapide de rôle pour l'acteur qui passe d'un personnage à un autre, dédoublement inévitable quand le nombre d'acteurs est limité à trois comme c'est le cas dans la tragédie grecque classique. 


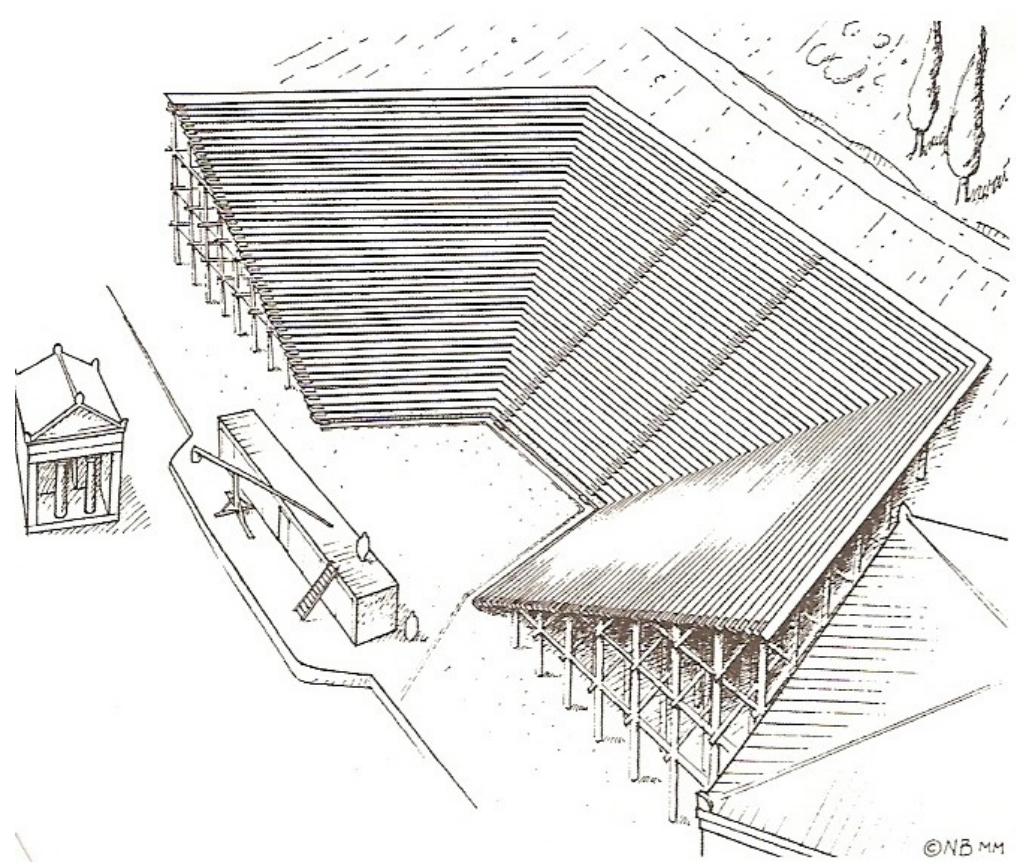

Le théâtre d'Athènes dans la seconde moitié du Ve siècle av. J.-C. : restitution, dessin de N.Bresh, extrait de J.C. Moretri, Théâtre et société dans la Grèce antique, Paris, 2001, p. 124.

L'espace dramatique tragique est donc un espace essentiellement fragmenté, et la communication entre ses différentes parties ne se fait pas aisément. Une double porte, au centre de la skénè, est le point de contact entre espace intérieur invisible et espace extérieur visible : elle reste la plupart du temps fermée, ne s'ouvrant que pour laisser un personnage sortir ou rentrer. Cette porte assure également une étanchéité sonore, une conversation extérieure ne pouvant être entendue à l'intérieur et réciproquement, à de rares exceptions près. Dans ces conditions, on constate une inévitable restriction des potentialités de jeu au profit des récits : chaque fois que l'action prend fictivement place dans un espace extra-scénique invisible, elle est non pas jouée mais relayée par un récit, élaboré avec le brio et le souffle de l'épopée, et auxquels les Grecs du $\mathrm{V}^{\mathrm{e}}$ siècle, grands amateurs de l'art de l'aède, devaient prendre un grand plaisir.

Cependant, les dramaturges de l'antiquité, qui étaient responsables de la production entière de leurs œuvres, de l'écriture à la distribution des rôles, de la composition de la musique à la chorégraphie, de l'instruction des citoyens formant les choeurs à la mise en scène, et parfois eux-mêmes acteurs dans certains de leurs drames, n'ont pas cessé de chercher à contourner les contraintes qui leur étaient imposées. Comment mieux équilibrer l'aspect visuel et l'aspect oral du spectacle? Comment franchir les frontières du visible ? Comment montrer de façon détournée ce qu'on ne doit pas montrer directement ? L'invention de l'eccyclème doit être lue comme un effort de dépassement du cloisonnement des espaces et des contraintes qui limitaient le jeu.

Décrivons ce qu'était cette machine scénique : sorte de plate-forme mobile, munie de roulettes, l'eccyclème permettait de faire « rouler » (ekkyklein, rouler à l'extérieur), hors du bâtiment de scène, par la porte centrale de la skénè, des personnages immobiles - parfois morts, parfois effondrés sous le coup d'un destin funeste. Deux sortes d'eccyclème apparaissent dans les textes critiques anciens (et notamment dans les scholies, utiles notes que les érudits alexandrins ajoutèrent dans leurs éditions des textes tragiques): l'une serait poussée sur la scène, après l'ouverture des portes, dans un mouvement de translation de 
l'intérieur vers l'extérieur ; l'autre aurait exhibé son contenu aux yeux du public en pivotant autour d'un axe central. Cependant, on admet généralement qu'au $\mathrm{V}^{\mathrm{e}}$ siècle seule la première structure a pu être utilisée, la seconde posant des difficultés techniques importantes (notamment l'ouverture de la double porte, plausiblement obstruée par un tel mécanisme).

\section{SCENERT AND MECHANICAL DEVICES}
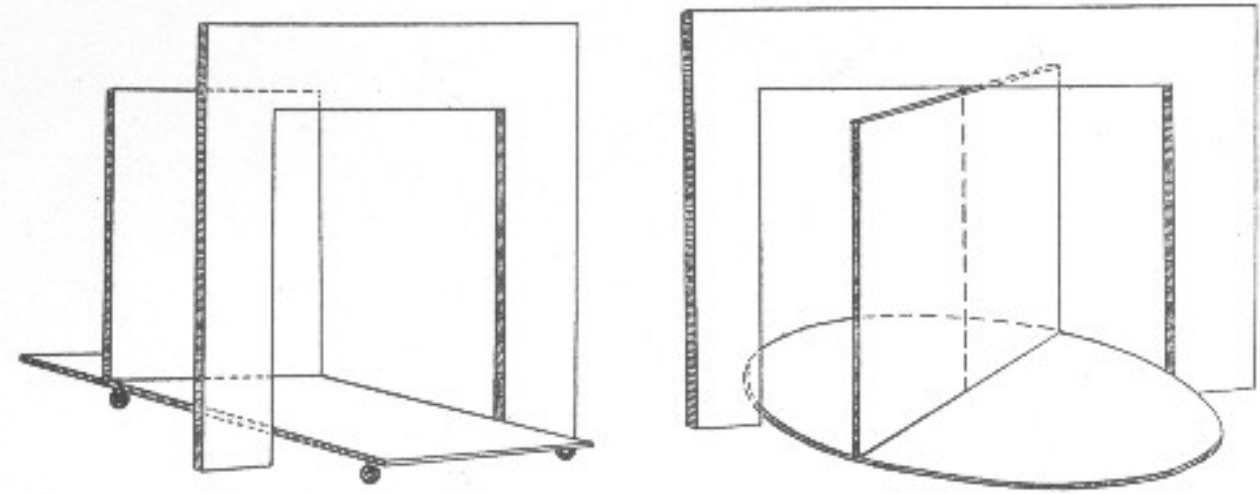

Fig. 280a-b. Possible forms of the eccyclema; Mahr

Schéma extrait de M. Bieber, The History of the Greek and Roman Theater, Princeton, $2^{\mathrm{e}}$ éd. 1961, p. 76.

Pour la faire sortir de la skénè, les acteurs eux-mêmes, ou bien un «technicien », à l'instar du mechanèpoios qui manoeuvrait la grue servant à l'apparition de divinités dans les airs, devaient pousser par l'arrière cette plate-forme, une fois les personnages installés. Nous ne disposons pas d'indice clair qui nous renseigne sur la vitesse à laquelle cette manipulation pouvait être effectuée, mais en tout cas, le mouvement obtenu par l'usage de l'eccyclème était probablement plus fluide et plus rapide que lorsqu'on utilisait des figurants qui apportaient les corps sur la scène. On peut supposer que, suivant les cas, les dramaturges pouvaient jouer sur la vitesse : faire mouvoir cette plate-forme avec lenteur et solennité ou bien la faire surgir sans effet d'annonce, pour saisir les spectateurs par la force et l'immédiateté de la vision. L'eccyclème, c'est donc une surface suspendue, surélevée par rapport au sol, où peut prendre place un groupe de personnages, qui se trouve propulsée hors de la skénè.

La seule preuve directe de l'existence de l'eccyclème au $\mathrm{V}^{\mathrm{e}}$ siècle av. J.-C. se trouve dans les œuvres d'Aristophane : à deux reprises, dans les Acharniens et les Thesmophories, des poètes tragiques devenus personnages de fiction, Euripide dans la première pièce, Agathon dans l'autre, surgissent sur la scène par le biais de l'eccyclème, explicitement désignée comme telle, dans un genre théâtral où toutes les ficelles du spectacle sont mises à nu.

DiCÉOPOLIS.

Euripide ! mon petit Euripide !

Réponds, s'il t'arrive jamais de répondre aux hommes.

C'est moi qui t'appelle, Dicéopolis de Cholléides !

EURIPIDE.

Non, je suis occupé. 
DicÉOPOLIS.

Alors fais-toi rouler sur l'eccyclème !

EURIPIDE.

C'est impossible.

DiCÉOPOLIS.

Fais-le quand même !

EURIPIDE.

D'accord, je vais prendre l'eccyclème ; je n'ai pas le temps de descendre ${ }^{l}$.

Dès l'antiquité, ce passage a été lu comme une parodie explicite des moyens dramaturgiques en usage dans la tragédie. Une scholie au vers 408 donne en effet une définition de l'engin : «Porte le nom d'eccyclème une machine en bois munie de roues, qui, en pivotant, montrait ce qui semblait se passer à l'intérieur, dans la maison, même à ceux qui étaient à l'extérieur, je veux dire aux spectateurs ${ }^{2}$. » Commentaires ajoutés plusieurs siècles après la création de l'œuvre, les scholies sont un matériau sujet à caution : ainsi la première partie de la définition, concernant le mécanisme de pivot, a été invalidée par les critiques modernes, comme nous l'avons déjà dit. On admet cependant la seconde partie sans discussion encore aujourd'hui. L'usage de l'eccyclème répond à un désir de montrer, au prix de la transgression momentanée de la frontière entre espace public et privé, visible et invisible, ce qui se passe à l'intérieur de l'oikos. Par convention - cette convention dont se moque un Aristophane soucieux de mettre en évidence l'artificialité de tels procédés -, la transition entre espace visible extérieur et espace invisible intérieur se fait par l'irruption de ce plateau mobile, morceau d'intérieur exhibé à l'extérieur pour la commodité de l'action. De fait, les textes des drames gardent eux-mêmes la trace de cette convention : les personnages présents sur l'eccyclème ou à côté, laissent entendre parfois qu'ils se trouvent toujours à l'intérieur de la maison ou du palais - «je me tiens là où j'ai frappé » affirme par exemple Clytemnestre, apparaissant sur l'eccyclème accompagnée des cadavres d'Agamemnon et de Cassandre.

La transition, c'est-à-dire le passage, d'un point de vue temporel comme spatial, d'une scène dramatique à une autre, est donc réduite à un mécanisme dramaturgique très rudimentaire, que les modernes ont parfois trouvé si exagérément conventionnel, et si impropre à préserver l'illusion théâtrale et la grandeur pleine de dignité du genre, qu'ils ont purement et simplement nié son existence au siècle de Périclès. Nous verrons que ceux qui l'acceptent, et avec raison, en considérant que certaines scènes des tragédies qui nous restent n'auraient pu être jouées sans l'eccyclème - sans parler de la nécessité de donner un sens aux scènes parodiques d'Aristophane que nous avons citées - tendent toutefois à réduire les potentialités scéniques offertes par cette structure scénique, en se soumettant à une définition conventionnelle qui appauvrit le discours critique.

Essayons en effet d'imaginer ce qui se passe quand l'eccyclème s'avance hors des portes de la skénè :

- Un espace autre que l'espace dramatique représenté par l'orchestra surgit et empiète sur la surface de ce dernier.

- Cette surface offre un espace détaché, suspendu, à part. Hormis les personnages qui s'y trouvent, c'est un espace le plus

\footnotetext{
${ }^{1}$ Aristophane, Acharniens, v. 404-408. Je traduis l'ensemble des passages cités, sauf indication contraire.

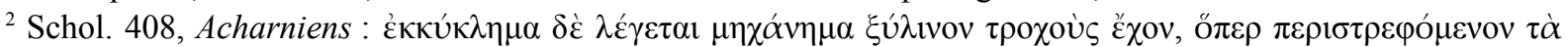

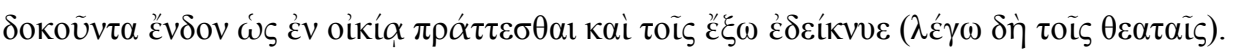


souvent vide, à première vue indéterminé, non marqué. Il est peut-être, à de rares occasions, caractérisé par des accessoires qui permettent d'identifier le lieu représenté.

- À l'opposition bipolaire entre skénè et orchestra, dedans et dehors, se substitue une relation à trois termes, l'eccyclème ouvrant un espace intermédiaire entre la skénè et l'orchestra.

Quelles peuvent être les différentes configurations de cette relation triangulaire ? Si l'eccyclème représente une parcelle d'intérieur, exhibée à l'extérieur par convention, quels rapports s'établissent entre les personnages se trouvant sur l'eccyclème et les personnages présents dans les autres espaces? Autrement dit, la question est de savoir si la juxtaposition des espaces fait transition ou conduit à la reconstitution d'une frontière, simplement déplacée sur les bords de la surface de l'eccyclème. Quelle communication est établie entre l'espace de la skénè, l'espace de l'eccyclème, et l'espace de l'orchestra? L'ambiguité de l'eccyclème, à la marge des espaces intérieur et extérieur, permet-elle un décloisonnement et une meilleure circulation, en brisant une frontière normalement étanche ?

Toute transition est un risque de rupture, de relâchement, d'affaiblissement de l'univers de la fiction théâtrale, le temps de passer d'un moment dramatique à un autre. L'art d'un metteur en scène consiste à en faire un usage signifiant, un temps lui-même dramatique et non détaché de la fiction. En considérant la transition spatiale opérée par le biais de l'eccyclème comme purement conventionnelle, et en limitant son usage à l'exhibition d'un espace intérieur dans l'orchestra, révélant un tableau montrant le résultat d'une action, les critiques du théâtre antique ont ignoré le plus souvent les multiples façons dont les dramaturges anciens se sont servis de cet expédient scénique pour reconfigurer l'espace dramatique et scénique. De plus, les poètes tragiques ont parfois accordé une signification dramatique et symbolique à cette machine, et cela le plus souvent en lien avec son identité physique et sa situation spatiale: le mouvement, le rythme, la position intermédiaire, ambivalente, suspendue de l'eccyclème peuvent suggérer le rejet hors du palais d'un personnage, son isolement ou bien la transgression que d'autres opèrent en brouillant les limites entre oikos et polis. C'est cet investissement tragique de la convention qui occupera notre réflexion : tantôt passerelle reliant les espaces, tantôt surface entrouvrant une faille entre eux, l'eccyclème est un outil dramaturgique protéiforme que chacun à leur tour, Eschyle, Sophocle et Euripide, ont exploité au service du sens tragique de leurs drames.

D'immenses zones d'ombre, non éclairées par les témoignages d'époque disparus, rendent notre connaissance de la dramaturgie antique sujette à caution : les poètes tragiques ne nous ont pas laissé d'arts poétiques qui éclaireraient leurs choix dramaturgiques, pas même de didascalies dans le texte de leurs drames, et nous n'avons pas davantage à notre disposition de comptes-rendus de spectacle rédigés par de passionnés spectateurs des concours tragiques... Le chercheur est donc sans cesse confronté à l'hypothétique, et les discussions critiques sur tel ou tel aspect du spectacle tragique ancien peuvent être développées à l'infini puisque aucune preuve décisive ne peut venir y mettre fin. Dans ces conditions, tout en m'appuyant autant que possible sur les témoignages les plus sûrs qui nous parviennent de l'antiquité, je m'accorderai le droit, une fois dépassé le stade des savoirs éprouvés, sûrs ou au moins hautement probables mis au jour au sujet de l'eccyclème, de proposer un point de vue personnel, argumenté mais subjectif, qui permettra, je l'espère, de réévaluer certaines idées préconçues ayant cours sur cet outil dramaturgique. 


\section{Eschyle : l'eccyclème avant l'eccyclème.}

L'apparition de l'eccyclème semble coïncider - dans les drames que nous avons conservés - avec l'apparition de la skénè au fond de l'orchestra, dans les deux premiers volets de la trilogie de l'Orestie ${ }^{3}$. Sitôt qu'est apparu ce hors-scène soustrait au regard des spectateurs, on aurait conçu le moyen de le rendre à leur vision panoptique ? Peut-être, mais nombre de critiques ne souscriraient pas cependant à une telle vision des choses : la nécessité de l'usage de l'eccyclème dans les scènes que nous allons exposer a été longuement discutée. Sous prétexte que l'eccyclème n'y est pas indispensable, on prétend que son usage n'est pas certain : certes, mais cela ne dit rien des potentialités dramatiques et dramaturgiques qu'elle offre.

Sont concernées les scènes d'exposition parallèles de cadavres qui marquent la fin d'Agamemnon (v.1372-1447), et des Choéphores (v. 973-1017): dans Agamemnon, Clytemnestre, l'épée à la main, apparaît sur l'eccyclème aux côtés des cadavres ; dans les Choéphores, Oreste de façon strictement symétrique, exhibe les corps de sa mère et d'Egisthe, en appelant le peuple d'Argos à y voir ceux qui sont les assassins de son père, avant d'être les victimes de sa propre vengeance.

Comme une scholie l'affirme ${ }^{4}$, on peut supposer que l'eccyclème fournissait ici un moyen commode de faire sortir les corps - représentés par les acteurs ou par des mannequins - dans un mouvement fluide. Nulle annonce de l'ouverture des portes ne se trouve dans le texte, comme il s'en trouve chez Sophocle et Euripide, annonces que 1'on a interprétées comme des signaux envoyés aux spectateurs pour les préparer à la sortie imminente de l'eccyclème. Loin d'être un argument en la défaveur de l'usage de l'eccyclème, cette absence de signal nous semble être l'indice de la recherche d'un effet de surprise, et peut-être en second lieu, d'un usage de l'eccyclème encore non normé, non établi dans des règles fixes. Sans céder au fantasme du primitivisme, nous voulons en effet voir dans ces scènes un usage premier de l'eccyclème, encore non défini par les contraintes de la règle, élaborée seulement dans un second temps : chez Eschyle, l'eccyclème permet le geste de l'exhibition triomphale, libéré de la nécessité de faire appel à des figurants, qui gâchent l'évidence de la vision. La nature de l'espace représenté sur l'eccyclème est floue, comme si cela importait finalement peu, alors que le mouvement et la situation réelle de l'eccyclème sont interprétés de façon métaphorique par les personnages qui en livrent une ekphrasis: Clytemnestre et Oreste présentent tous deux l'eccyclème comme l'image d'un lit dépravé par des amants adultères.

\section{Un espace intérieur ou extérieur?}

Elle est donc venue la revanche,

Enfin ! Et je me tiens là où j'ai frappé : cette fois, c'est fini.

J'ai tout fait, je ne le nierai pas,

Pour qu'il ne pût ni fuir ni écarter la mort ${ }^{5}$.

Presque incidemment, dans l'exultation de son crime, Clytemnestre donne une indication sur le lieu où elle se trouve, représenté par l'eccyclème : «je me tiens là où j'ai frappé », c'est-àdire à l'intérieur du palais, dans les appartements où elle a emmené Agamemnon, en feignant

\footnotetext{
${ }^{3}$ Nous n'étudierons pas en détail dans cet article la possibilité d'une utilisation de l'eccyclème au vers 64 des Euménides. Voir Peter Arnotт, Greek Scenic Conventions in the Fifth Century B. C., Oxford 1962, p. 82; Oliver TAPLIN, Stagecraft in Aeschylus, Oxford 1977, p. 442-443.

${ }^{4}$ Scholie au vers 973 des Choéphores : « la skénè est ouverte, et les corps apparaissent sur l'eccyclème ».

${ }^{5}$ Eschyle, Agamemnon (Ag.), 1378-1381.
} 
de vouloir lui prodiguer les soins d'une épouse dévouée. L'usage de l'eccyclème fait transition vers l'intérieur du palais.

Mais cet intérieur n'est pas découvert par les vieillards d'Argos, qui ont entendu les cris d'Agamemnon mais ont hésité trop longuement devant la porte du palais sans savoir que faire. Comme propulsé à l'extérieur, il s'impose à eux, sans qu'ils aient eu le temps de faire un geste. Cette immédiateté, et cette impuissance du chœur extérieur, le metteur en scène Peter Stein les a mises en scène en utilisant une eccyclème moderne construit sur le modèle de celle des Anciens, surgissant du palais et faisant reculer les vieillards horrifiés, sur le point d'ouvrir la porte pour porter secours à leur roi .

Dans les Choéphores on constate une évolution, puisque la fiction de l'intériorité de l'espace représenté sur l'eccyclème n'est pas maintenue. Alors qu'Oreste s'y trouve, il appelle le «père des hommes", le soleil, à contempler les assassins de son père :

Déployez-le vous-même. Approchez, et en cercle, montrez le voile qui enveloppa le héros, afin que le père voie, - non le père qui m'engendra, mais celui qui voit tout, le soleil ! - les forfaits infâmes de ma mère ${ }^{7}$ !

« Voyez », « Contemplez » tels sont les verbes qui reviennent sans cesse dans ses paroles : c'est le mouvement de l'exhibition au regard du peuple, au regard des dieux qui est en jeu. L'eccyclème n'est plus un espace intérieur, mais un espace de pur dévoilement.

\section{L'espace de l'adultère, miroir inversé du thalamos}

Dans leur désir de justification, Clytemnestre et Oreste proposent tous deux une interprétation du tableau macabre qu'ils ont mis eux-mêmes sous les yeux de leur public, une ekphrasis prenant les allures d'une argumentation voilée.

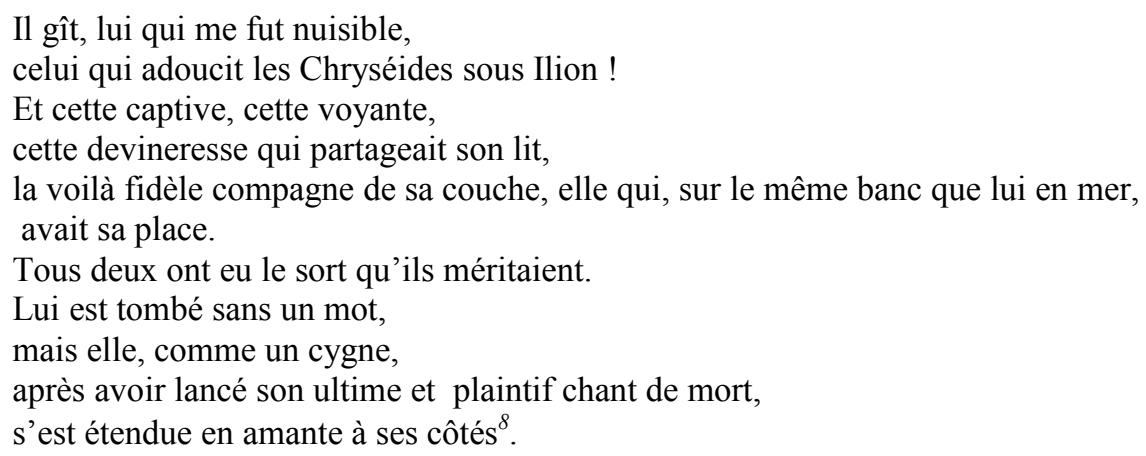

Après s'être présentée comme la vengeresse d'Iphigénie, Clytemnestre rappelle en montrant les corps des amants assassinés cette autre faute d'Agamemnon : avoir ramené de Troie une femme qui lui est une rivale. Dans l'interprétation de Clytemnestre, les amants gisent, amoureusement étendus l'un à côté de l'autre, comme s'ils partageaient sur l'eccyclème un lit de mort qui est aussi un lit nuptial (et peut-être étaient-ils de surcroît allongés non pas à même la surface de l'eccyclème, mais sur des lits qui étaient utilisés au

\footnotetext{
${ }^{6}$ Création de l'Orestie par Peter Stein à la Schaubühne de Berlin en 1980. Voir la critique de P. VAsseurLeGangneux, dans Les tragédies grecques sur la scène moderne, une utopie théâtrale, Presses universitaires du Septentrion, 2004, p. 104-105.

${ }^{7}$ Eschyle, Choéphores (Cho.), 982-986.

${ }^{8} \mathrm{Ag}$., 1438-1446.
} 
théâtre pour porter les morts). Funeste et horrible à voir, l'eccyclème est comme la perverse inversion du thalamos, la chambre nuptiale cachée au fond du palais, où les époux légitimes goûtent en toute liberté aux plaisirs de l'amour. Scandaleusement exhibés en dehors du palais, aux yeux du public, les amants Agamemnon et Cassandre jouissent dans la mort d'une union adultère obscène, mise en scène par Clytemnestre elle-même. C'est le topos des retrouvailles émues des époux dans l'intimité de la chambre, merveilleusement incarné par l'union finale d'Ulysse et de Pénélope dans l'Odyssée, qui se trouve ainsi en tous points outrageusement perverti.

Dans les Choéphores, Oreste se livre à une ekphrasis du même genre - ce qui va dans le sens d'une représentation symétrique de ces deux scènes parallèles - avec plus de pudeur cependant :

\footnotetext{
Voyez les deux tyrans de ce pays,

Les assassins de mon père, et les destructeurs de cette maison !

Vénérables, ils occupaient alors les trônes,

Compagnons tout comme aujourd'hui, autant que leurs maux

le laissent entendre, et leur serment demeure fidèle aux gages donnés.

Ensemble ils avaient juré de tuer mon misérable père,

et de mourir ensemble ; voilà qui est fait selon le serment ${ }^{9}$.
}

Oreste voit dans le rapprochement des cadavres, la fidélité jurée de Clytemnestre et d'Egisthe dans le meurtre et la mort reçue en commun, fidélité scandaleuse édifiée contre les liens du genos, qui attachent la femme au mari, le parent au parent.

Dans les discours de justification des meurtriers exhibant les dépouilles de leurs victimes, et en particulier dans celui de Clytemnestre, l'espace de l'eccyclème représente un thalamos profané par la lumière du jour et le sang des amants adultères. Sur la surface vide de l'eccyclème se superpose une image puissante, image de mots qui se déploie grâce à la simple disposition des corps, allongés sur l'eccyclème comme en un lit, un lit dévoyé, extrait du contexte normal de la chambre.

L'autre élément visuel marquant qui apparaît également dans les deux scènes, est le filet ensanglanté dans lequel Clytemnestre a pris au piège Agamemnon, et qu'Oreste exhibe de nouveau dans la scène d'exposition des cadavres des Choéphores comme rappel du meurtre de son père. Les corps enveloppés de cette toile rougie sortant du palais sur l'eccyclème sont un écho visuel aux tissus de pourpre que Clytemnestre avait fait déployer par ses servantes pour accueillir Agamemnon en grande pompe. Première image d'une coulée de sang s'échappant hors du palais, on pouvait y voir l'annonce du meurtre à venir. Les corps exhibés dans l'étoffe sanglante, rejetés hors de la skénè, comme si le palais dégorgeait en eux une souillure qui le condamne à jamais, apportent la confirmation de l'autodestruction en marche de la famille royale.

Dans ces drames d'Eschyle, l'usage de l'eccyclème semble être un usage d'avant la norme, même si la norme est peut-être déjà là en filigrane. On peut lire dans les scènes étudiées le désir d'une exploitation brute des caractéristiques de cette structure scénique au profit du spectacle, au profit de la monstration mise en scène par les personnages, sans qu'il y ait de scrupule concernant l'étrangeté ou l'artificialité de son procédé. L'eccyclème est désigné comme la projection d'un espace intérieur sur la scène dans Agamemnon, mais cette fiction n'est pas maintenue dans la seconde pièce : l'espace représenté sur l'eccyclème est instable. C'est en réalité les associations métaphoriques suscitées par le tableau qu'elle permet de montrer, qui jouent sur la double accointance de l'espace de l'eccyclème avec l'intérieur et l'extérieur, qui sont exploitées par le poète.

\footnotetext{
${ }^{9}$ Cho., 973-979.
} 
Dans une mise en scène récente de la trilogie eschyléenne, Olivier $\mathrm{Py}^{10}$ semble avoir tenté de traduire ce mouvement de projection des corps sur l'eccyclème. Réinterprétant le couple scénographique du théâtre antique skénè/eccyclème, il en fait les deux visages d'une même structure, un large cube de tôle métallique noire. Ce cube fait office de bâtiment de scène au début d'Agamemnon, puis après le meurtre perpétré par Clytemnestre, avance vers l'avant-scène en se retournant sur lui-même, laissant apparaître entre des murs d'une blancheur éblouissante les corps dévêtus, à peine recouverts du voile de pourpre, des acteurs jouant Agamemnon et Cassandre.

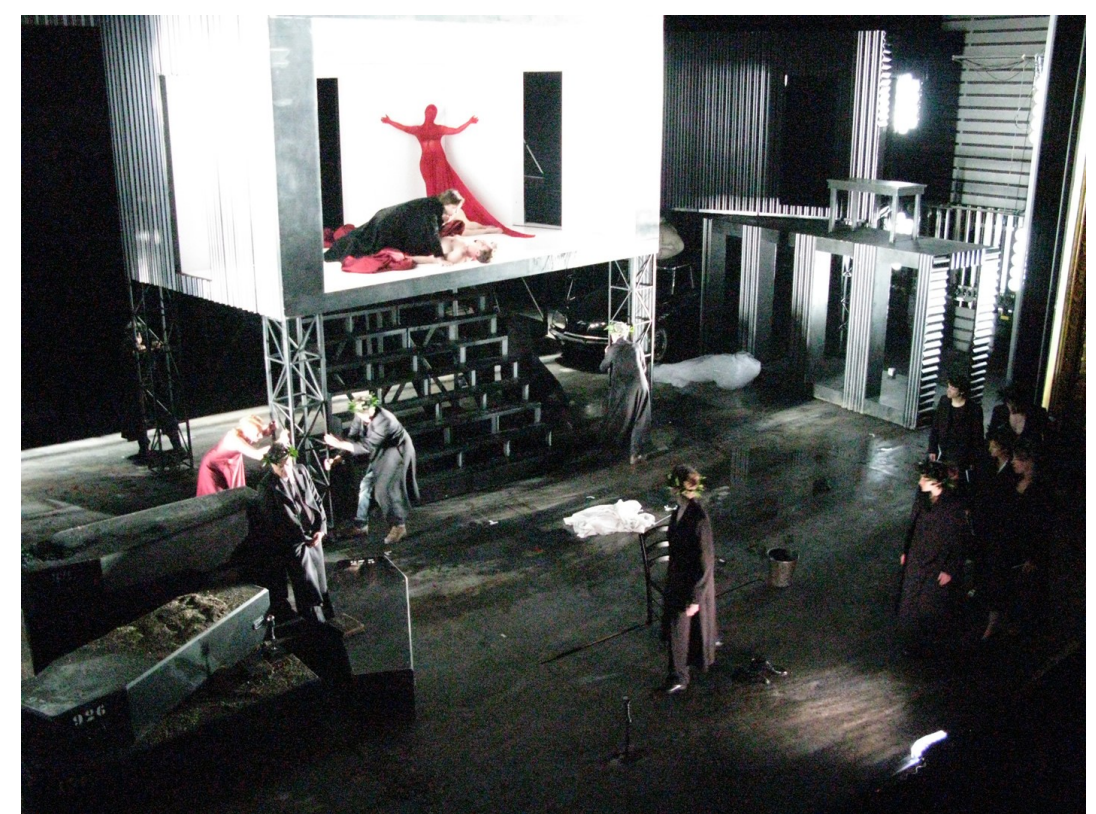

(C) photo Alain Fonteray - coll. Théâtre de l'Odéon - photo de répétition.

L'espace révélé par cette eccyclème moderne est étincelant, quand le plateau aux murs et au sol noir est envahi par l'ombre. Le contraste de lumière et de couleur marque la différence des espaces, et attire inévitablement le regard sur les corps des victimes, nus et intacts, comme saisis par la mort dans l'intimité de l'acte charnel - interprétation qui va dans le sens des propos de Clytemnestre dans le texte d'Eschyle. L'horreur et l'obscénité de ce tableau viennent alors du fait que l'intimité est profanée par la présence de Clytemnestre, d'Egisthe, d'une Iphigénie fantomatique conviée au spectacle qui consacre sa vengeance, et par le regard du public devenu voyeur.

\footnotetext{
${ }^{10}$ L'Orestie créé par Olivier Py au théâtre de l'Europe-Odéon, en juin 2008.
} 


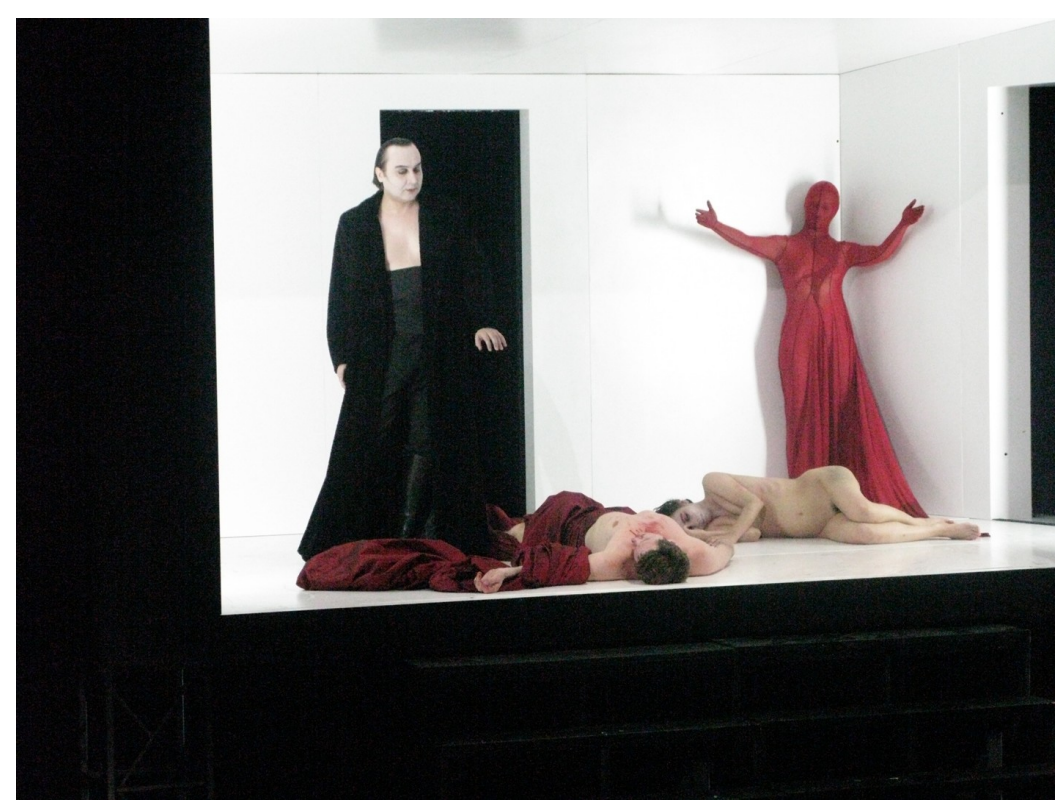

(C) photo Alain Fonteray - coll. Théâtre de l'Odéon - photo de répétition.

\section{Sophocle : extension du domaine des possibilités.}

Plus jeune de quelques années, Sophocle rivalisa avec Eschyle dans les concours théâtraux qui animaient les fêtes politiques et religieuses athéniennes: leurs pratiques théâtrales se sont certainement infléchies et enrichies au contact l'une de l'autre. Sophocle hérite probablement de l'eccyclème : il en développe les potentialités en jouant sur le double emploi possible de cette structure. Dans les pièces concernées, l'eccyclème est en effet exploitée comme engin mobile, permettant de faire sortir ou rentrer dans la skénè, dans un mouvement fluide et rapide, les personnages agissant; ou bien comme plate-forme offrant une surface de jeu intermédiaire entre la skénè et l'orchestra. L'un ou l'autre aspect est privilégié selon les cas, parfois les deux successivement : le mouvement rapide d'expulsion hors de la skénè ou de réintégration dans le bâtiment de scène prime ou bien la création d'un troisième espace de jeu.

\section{Un îlot d'intérieur exhibé à l'extérieur}

Conformément à la convention en usage, dans certains cas, l'eccyclème sert à assurer une transition rapide entre intérieur et extérieur, et à montrer sous la forme d'un tableau le résultat d'actions s'étant produites à l'intérieur. Mais si l'on s'en tient à cette description, on ne dit rien de ce qui se passe en termes de redistribution de l'espace. Nous verrons que l'irruption de l'eccyclème conduit parfois à repousser la limite entre intérieur et extérieur, tout en la renforçant, parfois à l'effacer. 


\section{La marginalité d'Ajax:}

Dans Ajax, pièce la plus ancienne conservée de Sophocle, la seconde entrée en scène du héros, assis au milieu des bêtes qu'il a sauvagement massacrées la nuit précédente, sous le coup de la folie insufflée par Athéna, est un des cas où l'utilisation de l'eccyclème paraît indiscutable. Dans un récit qui prépare les spectateurs au spectacle qu'ils vont avoir sous les yeux, Tecmesse, la compagne d'Ajax, décrit par avance l'état de prostration de celui-ci :

À présent, il gît dans cette infortune,

se refusant à manger et à boire, immobile,

tombé au milieu des bêtes qu'a frappées son fer ${ }^{l}$.

Les cris et les lamentations d'Ajax se font alors entendre de l'intérieur de la skénè, et le chœur demande à voir le héros :

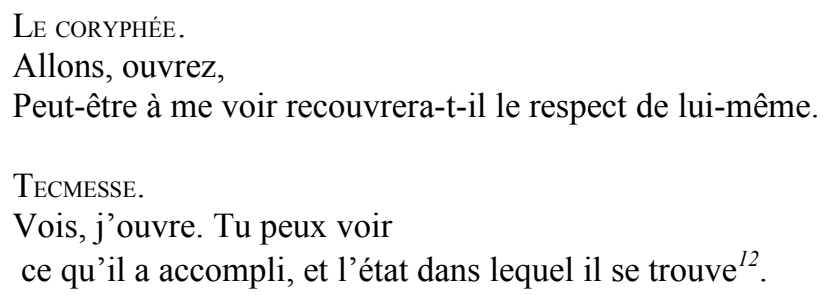

À ces mots, Tecmesse devait ouvrir les portes, et l'eccyclème s'avancer chargée de son contenu. La réaction pleine d'effroi du chœur laisse entendre qu'il voit tout du spectacle macabre. Un peu plus tard, le sang des victimes, susceptible d'effrayer l'enfant qu'Ajax réclame à ses côtés, est clairement mentionné dans le texte du drame ${ }^{13}$, et si l'on ne peut savoir par quoi les dépouilles animales étaient représentées, il semble raisonnable de supposer que l'acteur jouant Ajax, ainsi que la surface de l'eccyclème, étaient maculés de sang ou d'une substance s'en rapprochant visuellement. C'est l'immobilité d'Ajax et la présence fortement probable des dépouilles qui l'entourent qui imposent l'usage de l'eccyclème dans cette scène. Cette scène spectaculaire offre une « image qui concentre toute la tragédie du héros, où bourreau et victimes se confondent dans une même vision de sang et de destruction, qui est comme une annonce sinistre de la mort prochaine du héros ${ }^{14} \gg$. On ne saurait mieux dire.

Dans cet espace de mort, délimité par un cercle de sang, Ajax se trouve irrémédiablement séparé de sa compagne Tecmesse, et du chœur constitué par les marins qui l'ont accompagné à Troie. L'eccyclème est un espace détaché, et de part et d'autre de ses limites, aucune communication véritable ne s'établit. Cet aspect pouvait être traduit visuellement par le fait qu'aucun personnage ne monte sur l'eccyclème, à l'exception de l'enfant qu'Ajax appelle brièvement à ses côtés, le temps de lui transmettre ses dernières volontés. Par ailleurs, la parole se déploie de part et d'autre, sans qu'un dialogue se construise : comme cela a souvent été remarqué, Ajax ne se parle qu'à lui-même, et n'aspire désormais qu'à mourir tandis que tous l'exhortent à renoncer à un tel langage. Centré sur luimême, il commente avec amertume le sens nouveau que prend son nom à la lumière des événements produits : "Ajax », proche de l'exclamation douloureuse "aiai", équivalent grec

\footnotetext{
${ }^{11}$ Sophocle, $\operatorname{Ajax}(A j)$, v. 323-325.

${ }^{12} A j ., 345-347$.

${ }^{13} A j ., 545-547$.

14 Mauduit, Christine, Espace et dramaturgie dans le théâtre de Sophocle, monographie présentée en vue de l'habilitation à diriger des recherches, Paris IV-Sorbonne, 2000. p. 235.
} 
de l'interjection «hélas!", nom qui lui révèle à présent sa destinée. Sur l'eccyclème, la lamentation et l'introspection lui imposent une parole intransitive :

\author{
Ah, Ténèbres, ma lumière, \\ Erèbe pour moi plein d'éclat ! \\ Prenez-moi, prenez un nouvel habitant, \\ Prenez-moi ! Car ni vers la race des dieux, \\ ni vers celle, éphémère, des mortels, je ne suis digne \\ de tourner les regards pour obtenir de l'aide. \\ C'est la puissante déesse, fille de Zeus, \\ qui m'outrage mortellement ! \\ Où fuir ? Où partir et demeurer ? (...) \\ Que l'armée entière plutôt, une lance dans chaque main, \\ vienne me tuer ${ }^{15}$ !
}

Il n'y a plus de lieu possible pour Ajax en effet. Il ne saurait rester indéfiniment enfermé à l'intérieur de sa baraque, l'espace de l'oikos ne pouvant convenir à un héros de sa trempe. L'espace public lui est cependant désormais interdit car il n'y connaîtra plus que moqueries, injures si ce n'est violence meurtrière de la part de ses anciens compagnons d'armes. Sa position sur l'eccyclème, faille au milieu de l'espace de l'oikos et de l'espace de la polis, signifie visuellement cette impossibilité d'être dans quelque lieu que ce soit. L'eccyclème représente l'espace de l'exclusion, l'espace d'une vie déjà saisie par la mort, l'espace de la marginalité d'Ajax. Bien qu'elle permette de mettre en contact un morceau d'intérieur avec l'extérieur, elle n'assure pas de communication entre les espaces, mais renforce au contraire les frontières, en créant un endroit à part, où le héros peut évoluer... seulement pour un temps. Car cet îlot qui n'est que la négation des espaces qui lui préexistent, n'en est pas véritablement un, il n'est qu'une station sur le chemin du monde des morts, destination ultime du héros. A la fin de la scène, Ajax exhorte ainsi Tecmesse à refermer la porte sur lui :

\footnotetext{
Allons ! vite, prends l'enfant maintenant, verrouille notre porte, et cesse de pleurer devant la baraque. (...) Ferme la porte, tout de suite ${ }^{16}$ !
}

Selon Patricia Vasseur-Legangneux, «en règle générale, si nous nous fions aux tragédies conservées, seuls les cadavres sont roulés à l'intérieur de la skénè et retournent ainsi à la sphère privée ${ }^{17} . \gg$ En réalité Sophocle utilise à plusieurs reprises ce procédé consistant à faire rentrer sur l'eccyclème des personnages vivants à l'intérieur, comme nous le verrons plus avant; il n'en reste pas moins que cette sortie, si semblable à celle que l'on réserve aux morts, suggère une dernière fois la proximité d'Ajax avec eux.

\title{
Antigone : l'espace de l'oikos et de la cité unifiés sous le signe de la mort.
}

À la fin d'Antigone, les morts pleuvent et accablent l'une après l'autre Créon, le roi qui a fait trop peu de cas des lois divines et des liens du genos en condamnant Antigone à mourir. Celle-ci est suivie dans la mort par son fiancé Hémon, fils de Créon, et enfin par Eurydice, épouse de Créon, qui met en scène sa mort sur les autels domestiques du palais, tombant en victime sacrifiée au pouvoir dévoyé de Créon. Un messager sort alors du palais et

\footnotetext{
${ }^{15} \mathrm{Aj} .$, , 393-409.

${ }^{16} \mathrm{Aj} ., 578-581$.

${ }^{17}$ Patricia Legangneux-Vasseur, Ibid., p. 103.
} 
annonce à celui-ci, alors qu'il porte dans ses bras son fils immolé, qu'une autre mort l'attend dans le palais :

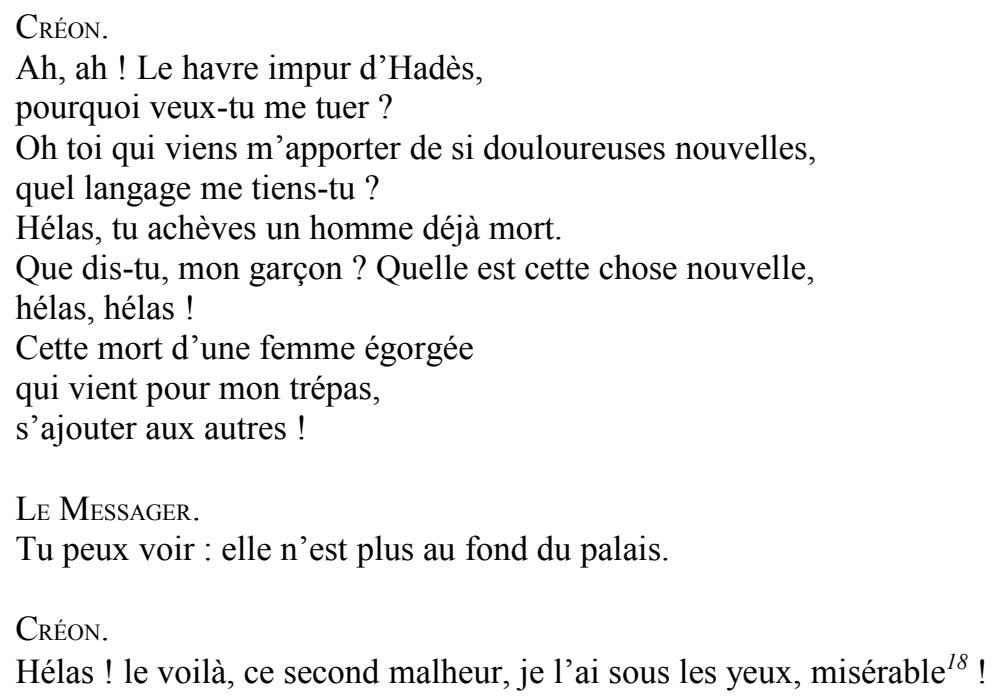

L'indication donnée par le messager sur la position du cadavre d'Eurydice («plus au fond du palais ») est un peu obscure, et les critiques s'interrogent par conséquent sur la manière dont son corps était montré, hésitant entre l'emploi de l'eccyclème ou la simple ouverture des portes, laissant apparaître le cadavre dans leur embrasure. C'est une question encore une fois indécidable ; cependant, il nous paraît intéressant d'évaluer la contribution de l'usage de l'eccyclème d'un point de vue scénique et dramatique. Cette dernière mort qui vient sanctionner l'injustice de Créon surgit de l'intérieur du palais et est identifiée comme une mort domestique, à la différence de la mort d'Antigone et de celle d'Hémon, advenues dans l'espace virtuel situé au-delà de l'orchestra. Avec la mort d'Eurydice, l'Hadès s'étend désormais à la totalité de l'espace. Comme l'écrit Jacques Jouanna « les cadavres convergent vers l'espace visible, venant d'abord de l'espace virtuel extérieur, puis de l'espace virtuel intérieur ${ }^{19}$. » À sa suite Christine Mauduit commente : «Avec cette image, qui est l'un des derniers tableaux de la tragédie, la mort envahit littéralement l'espace visible. Et la présence de ces témoins muets qui entourent Créon, l'atteignant en ce qu'il a de plus cher, dénonce aux yeux de tous la perversion d'un pouvoir qui, en faisant fi des droits sacrés du sang, a signé la ruine totale du foyer et du genos ${ }^{20}$. »

L'eccyclème nous semblerait justifiée ici par le fait que cette mort ultime est clairement désignée comme une mort domestique, sortant littéralement du palais, et d'aucuns ont d'ailleurs pensé que des accessoires, figurant les autels domestiques sur lesquels Eurydice s'est suicidée, pouvaient prendre place sur l'eccyclème à ses côtés, pour marquer clairement cet espace comme un espace intérieur. On peut aussi noter avec Christine Mauduit que « l'apparition du cadavre d'Eurydice, dans Antigone, joue (...) sur l'immédiateté et la surprise de la révélation ${ }^{21}$. " Cette dernière mort est en effet inattendue, le personnage d'Eurydice n'apparaissant pas toujours dans le cycle des Labdacides. L'utilisation de l'eccyclème serait tout à fait appropriée pour produire un effet de surprise.

Par ailleurs, le mouvement d'exhibition franc et direct du cadavre permis par l'eccyclème, serait un contrepoint tragique à la réserve auparavant montrée par le personnage

\footnotetext{
${ }^{18}$ Sophocle, Antigone (Ant.), v. 1284-1295.

${ }^{19}$ Jacques Jounnna, Sophocle, Paris 2007, p. 268.

${ }^{20}$ Christine Mauduit, Espace et dramaturgie dans le théâtre de Sophocle, monographie présentée en vue de l'habilitation à diriger des recherches, Paris IV-Sorbonne, 2000, p. 236.

${ }^{21}$ Christine Mauduit, Ibidem.
} 
d'Eurydice. Personnage secondaire, l'épouse de Créon apparaît brièvement, et son personnage est caractérisé par les scrupules qu'il exprime au sujet des convenances : sortir du palais est pour elle une audace - une femme est en effet à sa place à l'intérieur de la maison, et nullement dans l'espace public - qu'elle ne s'autorise que pour des motifs très convenables. Un retournement brutal se produirait : effacée de son vivant, Eurydice deviendrait dans la mort la démonstration criante du dévoiement du pouvoir de Créon.

À plusieurs égards, l'usage de l'eccyclème serait donc porteur de significations dramatiques et symboliques importantes. Cela permettrait en particulier de suggérer que l'espace dramatique tout entier est devenu territoire de mort, de l'oikos à la cité, jusqu'à l'espace extra-scénique virtuel de la grotte d'Antigone. Pour une fois, les différents espaces de la fiction ne sont désormais plus juxtaposés, mais fusionnent dans l'omniprésence de la mort.

\section{Toujours plus détaché.}

Dans Ajax, il est plausible que Sophocle ait utilisé l'eccyclème à plusieurs reprises, comme s'il avait tenté d'expérimenter dans un seul drame l'ensemble de ses potentialités scéniques et dramatiques. Dans la dernière scène où Ajax apparaît, la très célèbre scène de son suicide, une partie de la critique défend en effet l'utilisation de l'eccyclème pour des raisons techniques : l'eccyclème permettrait de soustraire in extremis aux yeux des spectateurs le spectacle du héros bondissant sur son épée. Sans rejeter ces raisons, nous préférerons tenter de justifier l'usage de l'eccyclème par des motifs d'ordre dramatique : à mesure que la pièce avance, l'isolement et l'éloignement d'Ajax du monde qui était auparavant le sien ne font que grandir. Lors de sa dernière apparition, l'eccyclème représenterait un espace toujours plus éloigné : dans la scène d'exhibition d'Ajax prostré au milieu des dépouilles, les limites de l'eccylème étaient infranchissables, et enfermaient Ajax dans une marginalité sans issue. Dans la scène de suicide, la signification portée par l'eccyclème évolue et approfondit le sème d'éloignement et d'exclusion, en atteignant l'espace de l'ailleurs sauvage, s'étendant au-delà des limites de la civilisation.

Exclu de la sphère privée comme de la sphère publique, Ajax décide de quitter le camp pour un lieu "vierge de pas humains », sur le rivage, annonçant à mots couverts son suicide à ses compagnons, qui ne saisissent nullement les sombres allusions présentes dans son discours. Ajax part donc comme on l'apprend par la suite, mais l'arrivée d'un messager révèle les menaces divines qui pèsent sur lui : selon les oracles du devin Calchas, si Ajax sort de chez lui ce jour-là, il trouvera la mort ${ }^{22}$. Sa vie dépend significativement du lieu dans lequel il se trouve, de la place qu'il occupe dans l'espace tendu par l'opposition entre intérieur et extérieur. Tous partent alors à sa recherche, y compris le chœur, en sortant de l'orchestra par les entrées latérales: se produit pendant un instant un événement rare dans la tragédie grecque, l'orchestra est vide. De cette façon, le dramaturge signifie le changement d'espace dramatique : quand Ajax surgit ensuite en effet, nous comprenons qu'il se trouve en dehors du camp, sur cette plage où il avait dit se rendre, et l'épée, prête à accomplir sa tâche, est déjà plantée en terre.

Le couteau du sacrifice se tient là, dressé de manière à trancher au mieux, si l'on peut avoir le loisir de calculer cela encore,

\footnotetext{
${ }^{22} A j .$, v. $750-755$.
} 
présent d'Hector, le mieux haï de mes ennemis, le plus odieux à ma vue.

Il est enfoncé dans la terre ennemie de la Troade,

fraîchement aiguisé à la pierre qui ronge le fer. (...)

Ainsi nous sommes prêts ${ }^{23}$.

La mise en scène de la mort d'Ajax a fait l'objet de longs débats. Tous les critiques reconnaissent l'audace de Sophocle, qui met à mal, plus qu'en aucun autre drame conservé, la séparation des choses pouvant être vues de celles qui ne se montrent pas sur la scène. Tous sont pour dire que le suicide du personnage était probablement montré, tout en étant dissimulé à la vue du public au moment paroxystique du bond, mais les divergences apparaissent au sujet des modalités de la réalisation technique de ce suicide à la fois visible et invisible. On peut dégager deux théories majeures des écrits consacrés à la mise en scène problématique de ce suicide: nous trouvons d'un côté les partisans de l'existence d'un panneau peint, représentant un buisson et se trouvant près de la porte de la skénè. Ce panneau dissimulait l'épée, qui n'était donc pas vue par les spectateurs, et au moment où Ajax bondissait, il était lui-même dissimulé par le panneau. L'acteur pouvait alors entrer dans la skénè sans être vu, se changer pour endosser un autre rôle et l'on pouvait en même temps mettre en place derrière le panneau un mannequin transpercé par l'épée, représentant le cadavre d'Ajax, découvert par la suite par Tecmesse derrière ce même buisson ${ }^{24}$. Ceux qui soutiennent cette proposition de mise en scène, reconnaissent généralement la nécessité de situer ce panneau peint à proximité des portes de la skénè, de façon à ce qu'une circulation soit possible pour l'acteur entre l'espace extérieur et l'espace intérieur.

Mais évidemment, l'eccyclème est une solution alternative également envisageable ${ }^{25}$, s'il s'agit d'assurer une communication entre l'espace de la skénè et l'espace extérieur et de soustraire rapidement à la vue des spectateurs le spectacle du héros s'empalant sur l'épée. Au début de la scène de suicide, selon cette hypothèse, Ajax se trouverait sur la plate-forme, sur laquelle serait également installée l'épée - dans les vers que nous avons cités, le texte semble en effet indiquer que tout est prêt dès que la scène commence. À la fin de sa tirade, l'eccyclème serait rentrée dans la skénè, le mouvement de retrait de l'espace scénique transposant métaphoriquement le saut dans la mort. Ainsi, quand le chœur entre en scène, l'espace de jeu serait vide de toute trace d'Ajax, le cadavre étant ensuite révélé aux yeux de tous sous la forme d'un mannequin, par une deuxième sortie de l'eccyclème (v. 891).

Cette proposition de mise en scène, non moins techniquement réalisable que la première, a le mérite de faire porter aux moyens techniques mis en jeu - soit l'eccyclème une signification dramatique et symbolique forte, et d'autre part de construire un parallèle intéressant avec le premier usage de l'eccyclème que nous avons commenté. Sans que cela puisse être un argument décisif en faveur d'une utilisation historique, il nous est possible de commenter les effets que produirait l'eccyclème dans ce cas précis.

En dehors de l'Ajax, on ne compte qu'une seule autre occurrence de changement de lieu scénique dans les textes tragiques qui nous sont parvenus : dans les Euménides d'Eschyle,

\footnotetext{
${ }^{23} A j ., 815-823$.

${ }^{24}$ C'est la mise en scène que propose par exemple Jacques Jounnna, Ibid., p. 254-255 : « il voyait Ajax bondir, mais dans le mouvement Ajax disparaissait derrière le panneau peint situé en face de la porte du bâtiment de scène. Les spectateurs ne voyaient donc pas l'épée plantée dans le sol dont parle Ajax dans son monologue avant le suicide ; elle est censée être cachée par le fourré. »(...) «Ainsi, sans cultiver le paradoxe, on peut affirmer que le suicide d'Ajax se déroule dans l'espace visible sans que l'instant décisif du suicide soit vu par les spectateurs. »

${ }^{25}$ David WiLes penche pour cette mise en scène, dont il décrit les effets dans Tragedy in Athens. Performance space and theatrical meaning, Cambridge 1997, p. 163-165. Voir également Peter W. MEINECK « Ancient drama illuminated by contemporary stagecraft: some thoughts on the use of mask and ekkyklema in Ariane Mnouchkine's Le dernier Caravansérail and Sophocles' Ajax », American Journal of Philology, 2006, vol 217, fasc 3, p. 455
} 
au moment où l'action dramatique se transporte de l'enceinte du temple d'Apollon à Delphes à l'Acropole d'Athènes, où Oreste est jugé pour son crime. Chez Eschyle comme chez Sophocle, le changement de lieu est accompagné de la sortie du chœur, signal scénique qui annonce aux spectateurs que l'action à venir se situe dans un espace différent. L'utilisation de l'eccyclème dans Ajax pourrait être un second signe émis à l'intention du public pour suggérer ce changement, et dans la continuité de son utilisation précédente, l'éloignement et l'isolement du héros. Dans ce lieu intact de toute présence humaine, sur ce rivage marin inviolé, Ajax est définitivement à l'écart de la société des hommes. Autrement dit, l'eccyclème assure un déplacement vers un espace extra-scénique qui n'est plus seulement celui de l'intérieur, mais il permet de relier désormais des espaces encore plus éloignés : l'espace de l'ailleurs non civilisé ou plus encore l'espace symbolique de l'exclusion et de l'isolement dans lequel le héros épique se trouve funestement confiné.

Peter Meineck suggère que Sophocle procèderait dans cette scène à un autre déplacement de l'usage conventionnel de l'eccyclème : la machine ne fait plus transition vers un espace intérieur physique, mais assure le passage vers l'intériorité, l'intimité d'Ajax, au moment où il se laisse saisir tel qu'il est, sans plus user du langage duplice qui le caractérisait précédemment ${ }^{26}$.

Dans tous les cas, la description conventionnelle de l'eccyclème ne suffirait pas à rendre compte d'un mécanisme transitionnel complexe, qui, chez Sophocle, relie des espaces de plus en plus éloignés, réels ou métaphoriques. De fait, la dernière transition symbolique effectuée par l'eccyclème, est le passage du monde des vivants au monde des morts.

\section{Le chariot d'Hadès.}

Les derniers mots d'Ajax sont d'émouvants adieux à la lumière :

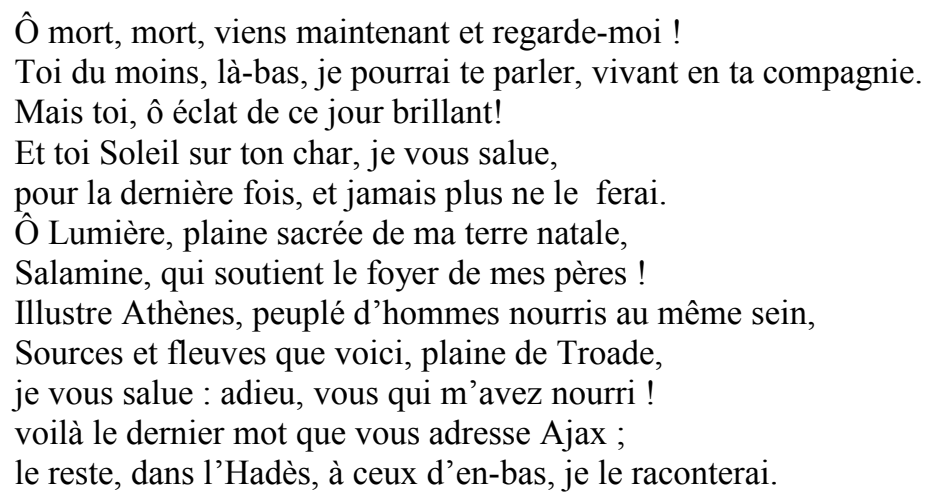

Si l'on soutient qu'Ajax se tient sur l'eccyclème, alors soustraite à la vue par un mouvement de retrait à l'intérieur de la skénè, alors Ajax quittant l'espace scénique voyait en réalité la lumière pour la dernière fois, avant d'être avalé par les ténèbres de l'espace intérieur. Les portes se refermant sur lui sont l'image des portes des Enfers, et la skénè la demeure d'Hadès. Le mouvement de l'eccyclème traduirait cet adieu à la lumière, et cette entrée dans le monde des ombres ${ }^{27}$.

\footnotetext{
${ }^{26}$ Peter W. Meineck, Ibid., p. 459 : «The Athenian audience understood that the ekkyklema was used to externalize the internal, to reveal what was hidden inside. Here, Sophocles goes one step further and allows his audience into the mind of Ajax at his most private and solitary moment; here the ekkyklema externalizes the internal mind. »

${ }^{27}$ voir l'analyse de David WiLES, Ibidem.
} 
Cette mise en scène nous paraît d'autant plus plausible que Sophocle semble avoir procédé de façon semblable à la fin de l'Electre. Au vers 1466, les critiques admettent généralement qu'Oreste et Pylade apparaissaient sur l'eccyclème, debout près du corps étendu de Clytemnestre, dont l'identité est dissimulée par un voile. Egisthe a en effet demandé à faire ouvrir les portes du palais, trompé par Electre, pensant savourer son triomphe en contemplant le corps sans vie d'Oreste ${ }^{28}$. Comme l'a remarqué Jacques Jouanna, malgré l'utilisation de l'eccyclème, la fiction de l'entrée d'Egisthe dans le palais n'est pas du tout entretenue, Egisthe et Oreste dialoguant comme s'ils étaient à l'extérieur; quand Oreste veut porter à Egisthe le coup fatal, il l'exhorte à entrer à l'intérieur ${ }^{29}$. La convention n'est pas du tout opérante pour décrire ce qui se passe dans cette scène : l'espace de l'eccyclème s'agrège à l'orchestra, tout en conservant un lien inquiétant avec l'intérieur du palais. Dans le dialogue rapide et tendu entre Egisthe et Oreste qui clôt la tragédie, les différents espaces voient leurs connotations requalifiées :

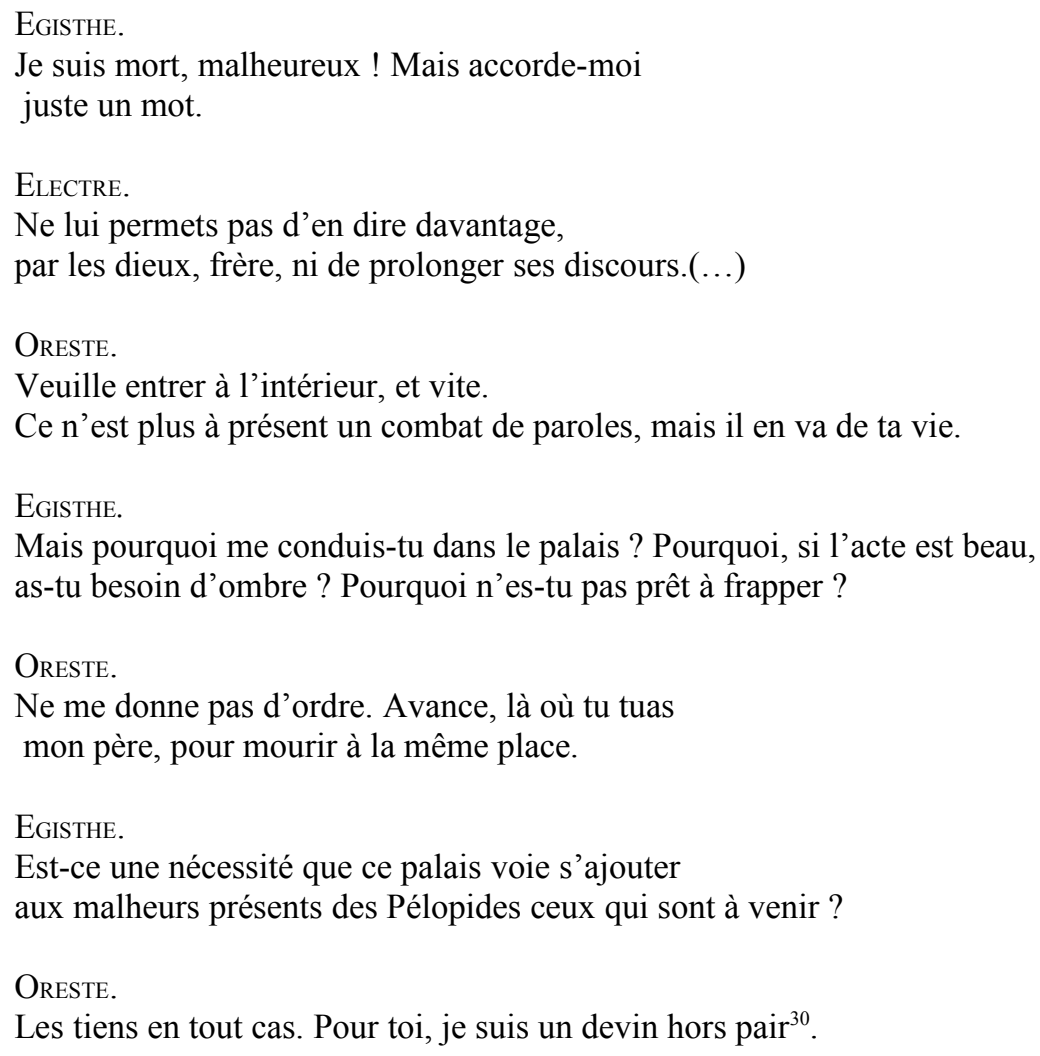

Bien entendu, faire entrer Egisthe à l'intérieur est une nécessité dramaturgique : sa mort ne peut avoir lieu dans l'espace scénique. Mais cette convention se trouve justifiée dramatiquement. Dans ce dialogue la skénè apparaît de manière négative, comme l'espace caché où la violence arbitraire a libre cours, à la différence de l'espace extérieur, connoté

\footnotetext{
${ }^{28}$ Sophocle, Electre (El.) , 1458-1465 :

Egisthe : J'ordonne qu'on se taise et que s'ouvrent les portes (...).

Electre : Voilà, je l'ai fait. Car avec le temps

je suis devenue raisonnable, et je m'accorde à mes maîtres.

${ }^{29}$ El., 1493-1494 : Egisthe s'étonne de devoir entrer « Mais pourquoi me conduis-tu dans le palais ? Pourquoi, si l'acte est beau, as-tu besoin de l'ombre ?»; v. 1502-1503: Oreste « - Toi, passe d'abord. » Egisthe « - Pour que je ne t'échappe pas ? ». cf Jacques Jounnna, Ibid., p. 269 : «cet échange de politesse sinistre est en désaccord avec la convention de l'eccyclème : comment Oreste peut-il précéder Egisthe en rentrant, puisqu'il est censé être à l'intérieur du palais ? Cependant le déroulement du spectacle est possible, malgré cette entorse à la convention. »

${ }^{30}$ El., 1482-1499.
} 
positivement en tant qu'il est public et démocratique. Le palais est par ailleurs le lieu où sont perpétrés les crimes qui déciment les Atrides depuis plusieurs générations, comme le rappelle Egisthe.

Si le meurtre d'Egisthe est évacué de l'espace scénique, et à double titre dans la mesure où il a lieu au-delà du temps du drame, cette scène de part et d'autre de l'eccyclème met l'accent sur le passage conflictuel entre espace du dehors et espace du dedans. On suppose qu'Egisthe passait alors devant l'eccyclème ou bien alors prenait place lui-même sur ce chariot d'Hadès. Le mouvement de retrait de l'eccyclème, à l'inverse du mouvement de sortie, entraîne la disparition inquiétante dans les ténèbres : c'est pour Egisthe un aller simple dans le monde des morts, effectué par un glissement rapide qui fait pendant au caractère pressant du dialogue échangé. L'eccyclème semble relier le monde des vivants au monde des morts, et la fluidité de son mouvement suggère à la fois l'implacabilité du sort et la brutalité d'Oreste. Celui-ci en reviendra, mais chargé de la souillure du crime et harcelé par les Erinyes.

Autant qu'il est possible de le supposer, l'eccyclème accomplit des fonctions variées dans le théâtre de Sophocle : son statut double de machine en mouvement, et de plate-forme offrant une surface de jeu alternative est exploité avec invention. L'eccyclème devient outil de transition reliant des espaces de plus en plus éloignés, réels ou symboliques, jusqu'à servir de véhicule faisant passer les héros du monde des vivants au monde des morts, procédé que le poète combine ingénieusement à la caractérisation de la skénè comme demeure d'Hadès.

\section{Euripide : variations virtuoses sur un thème connu.}

Comme ses prédécesseurs, Euripide a utilisé les moyens dramaturgiques en usage de son temps pour créer un espace dramatique en tension, et plus que tout autre, il semble avoir interrogé les conventions théâtrales qui préexistaient à son oeuvre, en tentant de les réformer quand elles lui paraissaient aller trop à l'encontre de l'illusion de réalité. Les occurrences probables de l'utilisation de l'eccyclème sont d'ailleurs assez limitées dans ses œuvres conservées (les critiques en comptent deux ou trois), et dans certains drames, on a noté la manière dont Euripide semble éviter l'usage de l'eccyclème en substituant à une scène d'exposition de cadavres attendue une action plus surprenante. C'est le cas par exemple à la fin de Médée : alors que Jason, bouleversé par la mort de sa jeune épouse et celle de son père, tente de forcer les portes du palais où se trouvent Médée et ses enfants, le coryphée lui annonce qu'il verra dans le palais un spectacle funeste :

Ouvre les portes : tu verras tes enfants égorgés ${ }^{31}$.

Une phrase semblable devait certainement sonner comme un signal annonçant la sortie prochaine de l'eccyclème hors de la skénè. Mais surprise, au même moment, Médée surgit dans le char du soleil, sur l'espace de jeu normalement réservé aux apparitions divines, le toit de la skénè, les cadavres de ses enfants à ses pieds. L'utilisation de l'eccyclème est évitée, au profit d'une séquence scénique imprévue et plus spectaculaire.

Euripide s'est néanmoins servi parfois de l'eccyclème : une occurrence plausible dans le drame perdu Cresphonte - et une occurrence certaine - dans Héraclès Furieux montrent la manière dont le dramaturge semble avoir travaillé la convention réglant l'usage de l'eccyclème, en l'appliquant de manière littérale ou en la poussant dans ses derniers

\footnotetext{
${ }^{31}$ Euripide, Médée, 1313.
} 
retranchements. Dans Cresphonte, nous verrons que l'eccyclème a pu servir pour mettre en scène dans l'espace visible une tentative de meurtre. Dans Héraclès furieux, tout se passe comme si le poète avait tenté de faire coïncider caractéristiques conventionnelles et caractéristiques réelles de l'espace de l'eccyclème, en faisant d'autre part de l'eccyclème non plus seulement l'outil de la transition spatiale mais sa fin propre.

\section{Cresphonte ou la focalisation sur l'espace intérieur.}

Le texte de Cresphonte, pièce jouée aux alentours de 425, nous est parvenu sous une forme très fragmentaire. Néanmoins trois sources littéraires anciennes assez précises quoique tardives par rapport à la date de la création de la pièce nous permettent de reconstituer son contenu, ainsi que d'imaginer certains aspects de sa mise en scène : un commentaire d'Aristote dans la Poétique, la fable 137 du latin Hygin, et un passage du traité De esu carnium de Plutarque ${ }^{32}$. Cette pièce raconte la manière dont Cresphonte retourne dans sa patrie, afin de tirer vengeance de l'usurpateur Polyphontès qui a assassiné les membres de sa famille et pris possession de sa mère. Selon les sources citées, un des moments pathétiques de ce drame advenait quand la mère de Cresphonte, ignorant son identité et le croyant l'assassin de son fils, s'apprêtait à le tuer d'un coup de hache, alors que celui-ci, ayant réussi à s'introduire par une ruse dans le palais, était endormi sur un lit. Un vieux serviteur s'interposait au dernier moment pour empêcher le crime, et à l'angoisse suscitée par l'appréhension d'un infanticide succédait pour le public la joie d'une émouvante scène de reconnaissance. Car si l'on en croit Plutarque, cette scène avait lieu sous les yeux du public, et serait restée dans les annales pour avoir provoqué le plus de frayeur dans les gradins:
« Regarde également cette Mérope bien connue dans la tragédie : comme meurtrière de son propre fils, elle lève la hache contre lui, en disant ' $<\ldots>$ ce coup que je te donne'. Quelle effervescence elle suscite dans le théâtre qu'elle fait sursauter de terreur dans la crainte qu'elle ne devance le vieillard qui veut l'arrêter, et ne blesse le jeune homme ${ }^{33}$. »

La question qui embarrasse alors les critiques est celle de la réalisation scénique d'une action supposée se passer à l'intérieur du palais ${ }^{34}$.

Euripide a mis en scène de manière récurrente des personnages endormis, malades ou encore mourants, reposant sur des lits, en en tirant des effets de pathétique ou bien de suspense. Cresphonte serait un cas parmi d'autres où un héros se trouve endormi dans l'espace scénique. Dans les passages comparables, l'apparition du lit dans l'espace scénique est toujours justifiée : Phèdre aura voulu prendre l'air malgré la faiblesse de son état, Oreste, chassé par tous, n'aura pas pu trouver un hôte qui l'accueille dans un lieu plus propre au repos. C'est pourtant déjà une façon de s'affranchir de la division stricte des espaces privés et publics, et d'un resserrement de l'intrigue tragique sur le destin individuel des héros. Dans Cresphonte, si la scène était explicitement située dans l'espace intérieur, une autre étape se

\footnotetext{
32 Aristote, Poétique, au sujet de la reconnaissance entre Mérope et Cresphonte : «Le meilleur cas est le dernier : dans Cresphonte (...) Mérope est sur le point de tuer son fils mais elle ne le tue pas et au contraire le reconnaît » (traduction F. Jouan et H. Van Looy, Euripide, Tragédies, tome VIII, Fragments, CUF 2002 p. 260). Hygin, Fable 137, « Mérope » : " Mérope croyant que celui qui dormait était le meurtrier de son fils, pénétra dans sa chambre munie d'une hache pour tuer son propre fils, sans s'en rendre compte, mais le vieillard le reconnut et fit obstacle au crime de la mère » (traduction F. Jouan et H. Van Looy, Ibid., p. 265) ; Plutarque, De esu carnium, II, 5, $998 \mathrm{E}$.

${ }^{33}$ Plutarque, De esu carnium, II, 5, 998 E, traduction F. Jounn et H. Van Looy, Ibid., p. 268.

${ }^{34}$ Voir par exemple A. HARDER, Euripides Kresphontes and Archelaos, Leiden, 1985, p. 115-117.
} 
trouvait franchie. Dans ces conditions, de tous les moyens proposés pour signifier scéniquement que l'action a bien lieu en réalité derrière les portes du palais ${ }^{35}$, en dépit des apparences, l'eccyclème paraît être l'outil dramaturgique le plus satisfaisant: aisément mobilisable, il ne risque pas d'autre part d'être mal interprété par le public. La présence d'un lit pouvait en outre servir de marqueur efficace pour suggérer visuellement l'identité du cadre de l'action.

À première vue, si l'eccyclème est utilisée pour effectuer une transition spatiale vers l'intérieur du palais, il n'y a rien là qui contreviendrait à la convention en usage. Cependant, si la règle est respectée, c'est pour mettre en scène une action qui devrait normalement se situer en dehors de la vue du public, puisqu'il s'agit d'un meurtre; d'autre part la fonction normale de l'eccyclème est de restituer à la vue des spectateurs une action accomplie en dehors de leur champ de vision, sous la forme condensée d'un tableau, et non pas l'action en train d'advenir. Dans ce cas précis, l'usage de l'eccyclème irait à l'encontre de la règle qui a présidé à son invention. Le dramaturge prend facétieusement à rebours la convention, sous couvert de s'y conformer, en mettant en scène sur l'eccyclème le meurtre sur le point de se faire.

Du point de vue de la distribution des espaces, il en résulte une focalisation sur l'espace intérieur, au détriment peut-être de l'espace extérieur, qui n'a plus rien à voir avec l'intrigue. Où pouvait se trouver le chœur, représentant de la collectivité populaire, pendant une telle scène, sinon en un endroit marginal, pour ne pas rompre la fiction d'une action prenant place dans un espace intime? La sortie de l'eccyclème ne fait pas seulement apparaître un espace domestique, elle opère un rapprochement, un mouvement de "zoom » sur l'intérieur et invalide sans doute momentanément la qualification de l'espace extérieur comme espace extérieur ouvert.

En détournant la distribution habituelle des espaces, par une utilisation probable de l'eccyclème, Euripide joue sur les attentes des spectateurs, d'une manière sans doute imprévue et nouvelle, qui n'a pas manqué de frapper les esprits. Le déclenchement de l'eccyclème n'opèrerait plus seulement un mouvement de translation d'une partie de l'intérieur à l'extérieur, mais un renversement de la configuration de l'espace s'accompagnant d'un brouillage des repères temporels (l'eccyclème surgissant non pas après mais avant le déroulement de l'action violente).

Héraclès furieux : la substitution de l'eccyclème à la skénè.

Quand la pièce commence, Héraclès descendu aux enfers pour chercher Cerbère passe pour mort, et Lycos, usurpateur du trône de Thèbes, décide de faire périr la famille de celuici : Mégara et ses enfants sont sur le point d'être immolés et perdent espoir malgré les encouragements du vieil Amphitryon, père d'Héraclès.

Contre toute attente, Héraclès revient des Enfers au deuxième épisode du drame, et tire vengeance de Lycos, en l'attirant à l'intérieur du palais. Se produit alors une nouvelle péripétie : l'apparition divine d'Iris et de Lyssa, déesse de la folie, prête à fondre sur Héraclès sur les ordres d'Héra. En effet un grand tumulte se fait entendre depuis l'extérieur, et le chœur décrit la manière dont le palais lui-même s'effondre ${ }^{36}$. Un messager en sort alors, et raconte

\footnotetext{
${ }^{35}$ A. HARDER (Ibidem.) recense les procédés suivants (en en acceptant aucun) : l'eccyclème ; un porche encadrant la porte de la skénè (élément de décor tel qu'il n'en est mentionné nulle part dans les tragédies classiques conservés, même s'il a été utilisé ultérieurement dans le théâtre hellénistique et le théâtre romain); le toit de la skénè ; ou la dissimulation de la scène à l'intérieur du palais, Cresphonte sortant à un moment par la porte, poursuivi par sa mère à l'extérieur.

${ }^{36}$ EuRIIIDE, Héraclès furieux (HF), 905 : « Voyez, voyez ! Un ouragan ébranle le palais, le toit s'écroule ! ».
} 
longuement et avec force détails comment Héraclès a sauvagement tué tous les membres de sa famille, avant d'être assommé par Athéna, et ligoté par son père Amphitryon. Apparaissent alors aux yeux du public Héraclès attaché à un tronçon de colonne, entouré des cadavres de ses enfants et de sa femme.

L'utilisation de l'eccyclème est unanimement reconnue à cet endroit, seul moyen dramaturgique de faire apparaître par la porte de la skénè des personnages immobiles. Défait et endormi, encerclé des dépouilles des membres de sa famille, Héraclès rappelle l'Ajax de Sophocle, mais à la différence de celui-ci, qui paraît alors qu'il a recouvré la raison, Héraclès est toujours dans un état d'inconscience, et le public sera spectateur de son retour progressif à la raison.

Les critiques se gardent généralement de définir la nature de l'espace représenté sur l'eccyclème. En effet, quand le réveil d'Héraclès se produit, ses premiers mots évoquent le soleil dont il sent les rayons :

\footnotetext{
Ah!

Je respire et j'ai sous les yeux le spectacle qui me convient,

Le ciel, la terre, et ces traits que darde le soleil ${ }^{37}$.
}

La situation réelle de l'eccyclème semble donc prendre le dessus sur la convention. Mais nous pouvons aller plus loin, en dépit des réserves émises par certains critiques sur la possibilité et l'intérêt de l'identification de l'espace ${ }^{38}$.

Un accessoire important se trouve sur l'eccyclème, si l'on en croit les didascalies internes : le tronçon de colonne auquel Héraclès se trouve attaché.

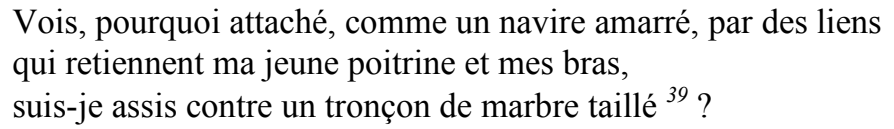

Le chœur a en effet assisté à l'effondrement du palais : cette colonne tronquée est la transposition scénique des ruines de l'édifice tout entier. Cet écroulement du palais comparable à celui que provoque dans les Bacchantes la puissance de Dionysos - devait certainement être un effondrement «verbal», mis en scène à travers les réactions de stupéfaction énergiques du chœur, mais laissé à l'imagination des spectateurs. On voit mal en effet comment cela aurait pu littéralement se produire sur la scène.

Quand l'eccyclème surgit, tout se passe comme si Héraclès apparaissait entre les ruines, synthétisées dans la présence de la colonne, et, on peut l'imaginer, de débris de pierres entre lesquels on pouvait distinguer trois corps d'enfants et un corps de femme, probablement représentés par des mannequins. Dans la suite de l'action, toutes les interactions des personnages ont lieu entre l'espace de l'eccyclème et l'espace de l'orchestra, la skénè étant condamnée par la présence de ce tableau en son point de contact avec l'espace extérieur. Elle n'a plus d'utilité dramaturgique, et nous inclinons à le penser, plus de fonction dramatique non plus. Le palais, qu'elle représente traditionnellement, y compris dans cette pièce, se trouve transféré sur l'espace de l'eccyclème, sous la forme qu'il adopte désormais. Par un effet de condensation et de synthèse, la plate-forme de l'eccyclème est désormais ce qui reste

\footnotetext{
${ }^{37} \mathrm{HF}, 1088-1090$.

${ }^{38}$ Voir par exemple Peter ArnotT, Greek Scenic Conventions in the Fifth Century B. C., Oxford 1962, p. 87: «To ask whether the tableau is supposed to be 'inside' or 'outside' only leads to confusion. It was a question which would never have troubled the Greeks. The ekkyklema was a convention partly dictated by practical difficulties, partly of value in itself as introducing effective tableaux at suitable moments of the drama (...). Obviously the ekkyklema shows what has been done inside the house ; but, once rolled out, it loses all sense of place and serves merely as a focal point for the ensuing action. »

${ }^{39}$ HF 1094-1097.
} 
du palais en ruines et en cela, le poète fait preuve d'une grande cohérence : si le palais s'est effondré, on ne peut plus se servir de la skénè comme si rien ne s'était produit. Un tronçon de colonne, quelques pierres dispersées sur l'eccyclème suffiront à suggérer les ruines, et par conséquent la skénè perd son sens et sa fonction dramatique transférés sur l'eccyclème jusqu'à la fin de la pièce. Elle se trouve bien sûr toujours là, à l'arrière-plan, mais se trouve comme désactivée, ne prenant plus part au drame, et n'étant plus éclairée par la lumière des mots - un spectateur moderne en tout cas n'aurait aucun mal à l'oublier, en se focalisant sur le nouvel espace de jeu qui est apparu.

Or, en assignant à l'eccyclème la fonction de représenter le palais détruit, Euripide dépasse la contradiction entre la situation réelle de l'eccyclème (à l'extérieur) et sa situation fictive (à l'intérieur) : en effet, un palais effondré est un espace lui-même ambivalent, un espace domestique fermé soudainement ouvert par la chute de ses murs, une demeure à ciel ouvert, envahie par les vents. Pour une fois, espace réel et espace dramatique coïncident, au prix, bien entendu, d'une condensation et d'une stylisation importantes, mais auxquelles les spectateurs anciens étaient habitués. Pour une fois aussi, l'eccyclème n'est plus seulement le médium de la transition spatiale, mais en est également le point d'arrivée. Ses caractéristiques physiques réelles sont exploitées et investies d'une fonction dramatique : représenter un état du palais, pendant toute la seconde partie de la pièce.

Portons enfin notre attention sur la manière dont les espaces communiquent, une fois cette transition effectuée: là encore, le jeu sur la norme est source d'une créativité remarquable. L'eccyclème, représentant les ruines du palais, est un espace ouvert à la lumière et aux vents, un espace troué, qui communique naturellement avec l'extérieur. Pourtant, dans les scènes qui suivent, une barrière difficilement franchissable semble s'ériger entre l'espace de la plate-forme, occupé par Héraclès, et le cadre extérieur qui l'entoure, l'espace de la cité. Jouant sur l'idée que l'eccyclème sert à exhiber des tableaux immobiles, le poète met en effet sous les yeux du spectateur un espace où les personnages sont figés dans la mort. Quand l'eccyclème sort de la skénè, rien ne bouge, Héraclès est endormi -peut-être mort, comme se le demande le choeur - et les corps des enfants et de Mégara à jamais privés de vie. Amphitryon intervient pour exhorter le chœur au silence: Héraclès n'est pas mort, mais endormi, le réveil pourrait entraîner un nouvel accès de délire ou alors lui faire quitter un état d'inconscience qui lui est un moment de rémission ${ }^{40}$ : le retour à la raison ne saurait lui apporter que douleurs atroces. Le temps semble donc s'arrêter dans ce tableau macabre, image du royaume des morts pour Héraclès qui se réveille ${ }^{41}$ ou encore champ de bataille anormal et terrible à voir ${ }^{42}$ pour Thésée qui arrive en scène à ce moment-là. Même quand il revient à la raison, Héraclès est comme figé à la vue du résultat de sa folie et pris lui-même dans une immobilité mortelle :

THÉSÉE.

Lève-toi, malheureux ! Tu as assez pleuré.

\footnotetext{
${ }^{40} \mathrm{HF}, 1054-1056$ : «Doucement, lamentez-vous sans crier, vieillards. S’il se réveille, il va rompre ses liens, tuer les citoyens, tuer son père et renverser le palais. » (trad. L. Parmentier, H. GréGoire, Euripide, Tragédies, tome III, CUF, 1950).

${ }^{41}$ HF 1101-1105 : « Je ne suis pourtant pas descendu une seconde fois dans l'Hadès, après avoir parcouru dans les deux sens la piste infernale, comme le voulait Eurysthée? Non, je n'aperçois ni le rocher de Sisyphe, ni Pluton, ni le sceptre de Déméter. Je suis frappé de stupeur ; je cherche en vain où je suis. » (trad. L. PARMENTIER, H. GRÉGOIRE, Ibid.).

${ }^{42} H F$ 1174-1177 : «Qui a tué ces enfants ? De qui la femme que je vois là est-elle l'épouse ? Il ne peut s'agir d'un combat; des enfants ne vont pas s'y exposer aux coups; je dois être en présence d'un malheur extraordinaire. » (trad. L. PARmentier, H. GrÉGoIre, Ibid.).
} 
HÉRACLÈs.

Je ne le pourrais pas ; mes membres sont figés.

THÉSÉE.

Car même les plus forts, les coups du sort les abattent.

HÉRACLÈs.

Hélas !

Puissé-je, pétrifié ici même, oublier mes maux ${ }^{43}$ !

On trouve là une sorte de variation virtuose sur le thème de la fixité du tableau, traditionnellement associées à l'utilisation de l'eccyclème : l'image de la pétrification semble à dessein faire allusion aux arts picturaux. Mais le poète use de ce procédé de façon à susciter une attente : que peut-il se passer si le temps s'arrête ainsi pour Héraclès pétrifié par la douleur? Est-ce la fin du drame, qui trouverait une fin pathétique dans l'image figée de l'auto-destruction de l'oikos, topos qui passe pour être à l'origine et à l'horizon de toute tragédie?

Justement non, cela ne s'arrête pas là, et Euripide élabore cette fausse fin pour surprendre le spectateur par une sortie qu'il n'attend pas. Héraclès furieux est en effet une pièce sur les liens de la philia, relation complexe fondée sur l'amitié et l'obligation de satisfaire à des devoirs envers la personne que l'on considère comme un philos. L'arrivée d'un médiateur, d'un passeur qui assure une liaison entre l'espace de mort représenté par l'eccyclème et la cité des hommes, en la personne de Thésée, offre à cette tragédie une fin « heureuse ». Il parvient à faire quitter à Héraclès l'espace de l'eccyclème, pour se remettre en mouvement, et regagner le monde des vivants, en rétablissant les liens de la philia que le meurtre d'Héraclès avait détruits. Ils sont figurés sur la scène par la main tendue par Thésée à Héraclès, pour l'aider à se redresser et lui offrir un soutien indéfectible : à l'issue de la tragédie, le héros tueur de monstres est un homme déchu, mais qui se tient debout grâce au bras de l'ami. Il parvient à se détacher du tableau, du groupe de statues dans lequel il figurait funestement, et franchit alors la frontière qui le séparait du monde des hommes en descendant de l'eccyclème.

À l'origine de l'eccyclème, une plate-forme à roulettes qui servait peut-être initialement à mettre en place et à enlever des objets lourds dans l'espace scénique, rendus ainsi plus aisément transportables. Puis les hommes de théâtre athéniens s'en sont emparés pour traduire en termes scéniques le geste de l'exhibition triomphale des dépouilles, après que le meurtre vers lequel la tragédie progresse a été accompli dans l'ombre du palais. À partir de là, le mouvement de la plate-forme de l'intérieur vers l'extérieur s'est trouvé investi d'une fonction de transition : les dramaturges ont utilisé l'eccyclème pour représenter dans l'espace scénique l'espace extra-scénique intérieur, comme un metteur en scène moderne peut jouer d'un éclairage, utiliser un rideau ou une toile peinte pour signifier un changement de lieu. L'outil a été réglementé par la formulation d'une norme, qu'auteurs et spectateurs connaissaient, et qui au départ répondait au besoin d'une univocité du signe assurant sa compréhension par le public : la transition vers un espace intérieur, et la condensation de l'action dans un tableau qui en montre le résultat.

Cependant nous avons voulu montrer que cette norme, transmise jusqu'à nous par des sources anciennes, fut sans doute très vite éclatée par les pratiques inventives des grands dramaturges classiques, et qu'elle ne doit pas être comprise comme une prescription suivie à

\footnotetext{
${ }^{43}$ HF 1394-1397.
} 
la lettre. Les drames des poètes tragiques montrent une recherche constante de nouveauté et de variété, et l'ensemble des potentialités scéniques de l'eccyclème ont été exploitées avec une grande souplesse, quelles qu'aient été les contraintes qui pesaient sur son usage. Objet simple, sans qualités, dénué de caractéristiques trop univoques, l'eccyclème devient, bien employée, un objet polysémique. Sur sa surface vide se logent les tensions qui nourrissent l'art tragique en profondeur: le rapport entre intérieur et extérieur, entre mouvement et immobilité, mais également entre le signe vu et sa signification qui le dépasse.

" Au théâtre, comme toujours, le public cadre, met le fleuve, les étoiles, le vent dans les arbres, la lune ou le miroitement d'une épée. Ça c'est le théâtre, c'est que nous [les metteurs en scène] donnons au fond le minimum essentiel. Et le public, nourri de cet essentiel, fait le reste, et devient le plus grand metteur en scène du monde. Au cinéma, j'ai l'impression que le public travaille moins, et qu'il faut le prendre plus par la main. L'espace ne se peuple pas de fantômes d'étoiles de la même manière, il peut aussi rester vraiment vide, vraiment prosaïque. Le bois peut rester du bois et ne pas devenir rivière. " Ces propos d'Ariane Mnouchkine, comparant le travail théâtral et cinématographique produit en parallèle sur Tambours sur la digue $e^{44}$, mettent bien en évidence la part des conventions, toujours à l'œuvre dans le théâtre moderne comme elle l'était dans le théâtre ancien, et l'effort corollaire que doit fournir le public pour suppléer en imagination à ce qu'on ne peut lui montrer sur la scène.

L'eccyclème était un outil de transition minimal entre des espaces parfois proches, parfois éloignés, parfois réels, parfois symboliques, et en effet les poètes tragiques confiaient au public la tâche de construire son sens, jamais donné a priori, mais sans cesse infléchi et édifié dans ses interactions avec les personnages ${ }^{45}$. Et de même qu'un effet de rideau ou d'éclairage peut avoir dans une mise en scène moderne une portée dramatique et symbolique qui dépasse son simple usage conventionnel, de même, l'eccyclème fut un médium transitionnel investi d'un contenu tragique riche et varié.

Anne-Sophie Noel

AMN à l'Université Jean Moulin - Lyon 3. eiposenna@wanadoo.fr

\section{Bibliographie}

Mazon P., Eschyle, Paris, CUF, 2 vol., 1e éd 1921 et 1925, $11^{\mathrm{e}}$ tir. 1976 et 1983.

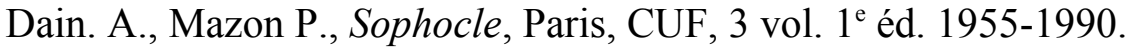

Méridier L, Parmentier L., Grégoire H., Jouan F., Euripide, Paris, CUF, 8 vol. 1926-1983.

Coulon V., Van Daele H., Aristophane, Paris, CUF, 5 vol., 1éd. 1923-1930.

Arnott, Pieter, Greek Scenic Conventions in the Fifth Century B. C., Oxford 1962.

Bieber, Margaret, The History of the Greek and Roman Theater, Princeton, $2^{\mathrm{e}}$ éd. 1961.

\footnotetext{
${ }^{44}$ Ariane Mnouchrine, Tambours sur la digue, coproduction Théâtre du Soleil, ARTE France, Bel Air Media, 2002.

${ }^{45}$ Jacques JounnNa fait ce constat: « «l'un des paradoxes du texte écrit par les tragiques grecs est que, tout en ayant été composé pour une représentation unique, il renferme des indications sur le spectacle comme si elles étaient destinées à guider un metteur en scène pour une nouvelle représentation, ou un lecteur pour imaginer le spectacle.» (Ibid., p. 254). Cela est vrai, mais selon nous, ces indications sont avant tout destinés aux spectateurs, qui, à chaque représentation, se voient donner les clefs de lecture nécessaires pour comprendre un spectacle qui évolue et se transforme sans cesse d'une pièce à l'autre.
} 
Dale, Anne Mary, «Seen and unseen on the Greek stage : a study in scenic conventions », «Interior scenes and illusion in Greek drama », in Collected Papers, Cambridge 1969.

Jacques, Jean-Marie, «La comédie nouvelle a-t-elle utilisé l'eccyclème ? », Actes du Colloque Où courir? Organisation et symbolique de l'espace dans la comédie antique, Pallas, 54, 2000, pp. 89-102.

Jouanna, Jacques, Sophocle, Paris, 2007.

Legangneux-Vasseur, Patricia, Les tragédies grecques sur la scène moderne, une utopie théâtrale, Presses universitaires du Septentrion, 2004.

Mauduit, Christine, Espace et dramaturgie dans le théâtre de Sophocle, monographie présentée en vue de l'habilitation à diriger des recherches, Paris IV-Sorbonne, 2000.

"À la porte de la comédie ", Actes du Colloque Où courir ? Organisation et symbolique de l'espace dans la comédie antique, Pallas, 54, 2000, pp. 25-40.

Meineck P.W., «Ancient drama illuminated by contemporary stagecraft : some thoughts on the use of mask and ekkyklema in Ariane Mnouchkine's Le dernier Caravansérail and Sophocles' Ajax », American Journal of Philology, 2006, vol 217, fasc 3.

Moretti, Jean-Charles, Théâtre et société dans la Grèce antique, Paris, 2001.

Pickard-Cambridge, Arthur, The theatre of Dionysus in Athens, Oxford 1956 (p. 100-122, « The ekkuklema »)

Scullion, Scott, Three studies in Athenian dramaturgy, Stuttgart/Leipzig, 1994.

Taplin, Oliver, - Greek Tragedy in Action, Londres, 1978.

Stagecraft in Aeschylus, Oxford, 1977.

Wiles, David, Tragedy in Athens. Performance space and theatrical meaning, Cambridge, 1997.

\section{Références de l'article :}

Anne-Sophie Noel, «Eccyclème et transition spatiale dans le théâtre tragique grec du V siècle av. J.C. ", Agôn [En ligne], N 1 : Interstices, entractes et transitions, Dossiers, Dramaturgies de l'interstice, mis à jour le : 05/12/2008, URL : http://agon.ens-lsh.fr/index.php?id=710. 